\title{
(3)
}

\section{Study of the Validity of Neutrophil CD64 and Serum Procalcitonin as Diagnostic Markers to Discriminate Infection from Disease Activity in Patients with Systemic Lupus Erythematosus}

\author{
Ahmed Ragheb ${ }^{1, *}$, Ahmed A. Sonbol ${ }^{2}$ \\ ${ }^{1}$ Internal Medicine Department, Faculty of Medicine, Menoufia University, Shebin Elkom, Menoufia, Egypt \\ ${ }^{2}$ Clinical Pathology Department, Faculty of Medicine, Menoufia University, Shebin Elkom, Menoufia, Egypt
}

\section{Email address:}

ahmed_ragheb76@yahoo.com (A. Ragheb), ahmed_sonbolmmm@yahoo.com (A. A. Sonbol)

\section{To cite this article:}

Ahmed Ragheb, Ahmed A. Sonbol. Study of the Validity of Neutrophil CD64 and Serum Procalcitonin as Diagnostic Markers to Discriminate Infection from Disease Activity in Patients with Systemic Lupus Erythematosus. American Journal of Clinical and Experimental Medicine. Vol. 3, No. 3, 2015, pp. 110-117. doi: 10.11648/j.ajcem.20150303.17

\begin{abstract}
Introduction: In addition to the complexity of the clinical presentation of both infections and disease activity in systemic lupus erythematosus (SLE) patients, the difficulty in making the therapeutic decision require investigations that should be of diagnostic value. Neutrophil CD64 is up regulated within few hours in patients with infection. Similarly, serum procalcitonin (PCT) levels increase rapidly following bacterial infection. Objective: The aim of this work is to study the usefulness of neutrophil CD64 expression and serum PCT as diagnostic markers to discriminate infection from disease activity in patients with systemic lupus erythematosus. Methods: This study was carried on 20 healthy females as controls (group I) and 55 female patients with SLE. Patients were distributed as following; 20 SLE patients without activity or infections (group II), 20 SLE patients with lupus activity (group III), and 15 SLE patients with infection (group IV). CBC, ANA, Anti-ds DNA, C3 and C4 were measured in all population. Serum PCT was measured by ELFA and neutrophil CD64 expression was done by flowcytometry. Results: Neutrophil CD64 expression and serum PCT levels were increased significantly in SLE patients with infection compared to those with disease activity. We demonstrated significant correlations between CD64 and markers of both activity and infection, while serum PCT levels were significantly correlated with markers of infection. The area under the ROC curves for detection of infection (AUC; 95\% CI) for neutrophil CD64 expression and serum PCT were (0.90; 0.79-1.01) and (0.99; 0.95-1.01), respectively. Conclusion: Our findings can prove that both neutrophil CD64 and serum PCT are reliable markers to discriminate infection from disease activity in SLE patients. Serum PCT was more accurate than neutrophil CD64 expression.
\end{abstract}

Keywords: Systemic Lupus Erythematosus, Lupus Activity, Infection, CD64, Procalcitonin

\section{Introduction}

Systemic Lupus Erythematosus (SLE) is a complex autoimmune disease with heterogeneity in clinical manifestations and disease course, characterized by pathogenic autoantibody formation, immune complex deposition, and end-organ damage. Despite the fact that the mortality and morbidity of patients with SLE has improved significantly during the last few decades, mortality rates remain approximately three times those of the age-matched and sex-matched population in most studies [1].

The complexity of the clinical presentation of both infections and disease activity in SLE patients requires investigations that may be of diagnostic value. Unfortunately, common screening laboratory tests employed to diagnose infections such as leukocyte numbers, presence of immature forms, CRP and ESR have poor sensitivity and specificity. Culture results are often viewed as confirmatory, but in practice they are often not used in treatment decisions because of their relatively slow turnaround times of up to 72 hours or more [2]. Due to the major differences in treating the two different situations, activity or infection, a rapid diagnosis is mandatory. Physicians are therefore always on the lookout for a method that would be helpful in the difficult decision as to 
whether to initiate antibacterial therapy or not [3].

Neutrophil granulocytes express their Fc $\gamma$ receptor 1 (CD64) antigen, predominantly when they are activated. CD64 is a type of integral membrane glycoproteins known as Fc $\gamma$ receptor 1 that binds monomeric IgG with high affinity [4]. Expression of the CD64 antigen on neutrophils has been under investigations for some years as a biomarker of infection and sepsis. On resting neutrophils, CD64 expression is low and after activation it is significantly up regulated within few hours. CD64 expression represents a physiological process which plays a key role in the innate immune response [5].

Procalcitonin (PCT) is a precursor of calcitonin that is primarily produced by parafollicular or calcitonin cells of the thyroid gland. Serum PCT is normally undetectable $(<0.05$ $\mathrm{ng} / \mathrm{ml}$ ) in healthy individuals, but its level increases rapidly following bacterial infection [6]. In contrast to CRP, expression of PCT is not elevated following non-infectious inflammation or non-bacterial infections [7-9].

\section{Methods}

The protocol for this study followed the ethical standards and was approved by the ethical committee of our institution and all subjects gave informed consent to participate in this study. The present study was conducted as a case control study on all SLE patients $(n=55)$ who were registered at the inpatient department and SLE special outpatient clinic in Menoufia University Hospitals from June 2013 to June 2014. Patients were distributed as following; group II: included 20 SLE patients without activity or infections (patients attending the outpatient clinic for follow up), group III: included 20 SLE patients with lupus activity and group IV: included 15 SLE patients with infection. In addition, 20 normal females were included as controls in group I. They were defined by self-report and confirmed by physician observations. All subjects underwent full history taking and complete physical examination. Diagnosis of SLE (in groups II, III and IV) was done according to the 1982 revised criteria for the classification of systemic lupus erythematosus [10] updated in 1997 [11]. Diagnosis of SLE activity (in group III) was according to the 2002 modified Systemic Lupus Erythematosus Disease Activity Index score (SLEDAI-2K) [12]. SLEDAI score change $>12$ was required to diagnose severe activity. Diagnosis of infection (in group IV) was depending on pathogen proven by microbiologic culture [13].

\subsection{Laboratory Assessment}

All samples from patients with infections were taken before antibiotic therapy was initiated. Five $\mathrm{ml}$ of venous blood were collected from each subject by venipuncture via a plastic disposable syringe and under complete aseptic conditions. $1.5 \mathrm{ml}$ of venous blood was transferred into a clean K-EDTA tube for complete blood count and CD64 measurement on neutrophils. $1.6 \mathrm{ml}$ of venous blood was transferred into a clean glass tube containing $0.4 \mathrm{ml} \mathrm{Na}$ citrate used for erythrocyte sedimentation rate. The remainder of venous blood was transferred into a plain tube for serum PCT, C-reactive protein, ANA, anti-ds DNA and $\mathrm{C} 3$ and $\mathrm{C} 4$ determination. Bacteriological samplings (sputum, throat and skin swab \& urine) were cultured on blood agar, chocolate agar and MacConkey's medium according to type of sample.

CBC was measured by Advia 2120 automated blood counter (Siemens -Tarrytown, NY-10591-USA), Erythrocyte sedimentation rate (ESR) was measured by Westergren method [14] and C-reactive protein (CRP) was measured by slide agglutination method [15].

Anti-nuclear antibody (ANA) was measured by indirect immunofluorescence assay [16]. Anti-double stranded DNA (Anti-ds DNA) was measured by ELISA [17]. Complement components 3 and 4 (C3 and $\mathrm{C} 3$ ) assays were done by ELISA [18].

\subsubsection{Measurement of the Fc Gamma Receptors 1 (CD64)}

Flow cytometric determination of neutrophil CD64 expression was performed. Samples were processed within 24 hours of collection, and whole blood samples were diluted with PBS. For each sample, two tubes were prepared (one for test and the other for the isotypic control); $100 \mu \mathrm{l}$ of the diluted sample was added to $10 \mu \mathrm{l}$ MoAbs (MCA756F) mouse antihuman CD64 : FITC (abcam, Cambridge, MA, USA), vortexed, and incubated at room temperature for 15 min in the dark. Three milliliters of the Lyse solution was added to each tube, vortexed, and then incubated for $15 \mathrm{~min}$ at room temperature. The tubes were centrifuged at $3000 \mathrm{rpm}$ for 5 minutes, and then the supernatant was discarded. The cells were washed twice with $2 \mathrm{ml}$ PBS, with repeating centrifugation, followed by discarding of the supernatant [19]. Samples were then ready for processing on the flow cytometer. Data were acquired on a FACS caliber flowcytometer (Becton Dickinson immune cytometry systems, San Jose, CA, USA). The instrument set up was checked weekly using QC windows beads (flowcytometry standard, San Juan, PR). Results were expressed as percentages of neutrophils subset positive for CD64 marker and their mean fluorescence intensity [20].

\subsubsection{Measurement of Serum PCT}

Serum procalcitonin (PCT) was done by VIDAS $\AA$ $\mathrm{B} \cdot \mathrm{R} \cdot \mathrm{A} \cdot \mathrm{H} \cdot \mathrm{M} \cdot \mathrm{S}$ PCT (bioMérieux, Marcy l'Etoile, France) which is an automated enzyme-linked fluorescent immunoassay (ELFA) based on a one-step immunoassay sandwich method and a final fluorescent detection step for the quantitative measurement of PCT. This immunoassay is performed on the mini VIDAS systems which are fully adapted for emergency conditions [21].

Two ready-to-use reagents; The SPR ${ }^{\circledR}$ Solid Phase Receptacle serves as a solid phase and pipetting device, coated with mouse monoclonal anti-human procalcitonin immunoglobulins and the reagent strip contains predispensed reagents.

-The reaction occurs within the interior of the SPR whereby anti-calcitonin antibodies and conjugate form a sandwich. 
-4-MUP is cycled into SPR and conjugate enzyme catalyses the hydrolysis of the substrate into 4-Methylumbelliferone which is measured at $450 \mathrm{~nm}$.

Measuring Range: 0.09-200 ng/mL. Quality Control: Two controls are included in the VIDAS B $\cdot \mathrm{R} \cdot \mathrm{A} \cdot \mathrm{H} \cdot \mathrm{M} \cdot \mathrm{S}$ PCT kit to ensure the quality of the results obtained. Calibration: Is performed by using two calibrators ( $\mathrm{S} 1$ and $\mathrm{S} 2$ ) included in the kit and is performed when a new lot of reagents is opened and then once every 28 days. This operation provides instrument-specific calibration curves and compensates for possible minor variations in assay signal throughout the shelf-life of the kit [22].

\subsection{Statistical Analysis}

We used the statistical package of social signs (SPSS, version 16) to perform the analysis. Categorical data were presented as number and percentages and continuous variables as means \pm standard deviation (SD). One way ANOVA test or Kruskal-Wallis test were used as appropriate for comparison of quantitative variables more than two independent groups. Intergroup comparisons were performed using the chi-square test, t-test, and Mann-Whitney U test as appropriate. Pearson's correlation coefficient, $r$, was used to assess the relationship between CD64 and other variables in the three patient groups. Receiver operator characteristic (ROC) curve was used to determine cut off values of both neutrophil CD64 expression and serum PCT with optimum sensitivity and specificity in diagnosis of infection (i.e. between groups 3 and 4).

\section{Results}

Fifteen female SLE patients with infection were included in group IV. Five patients $(33.3 \%)$ had chest infections (bronchopneumonia), two patients (13.3\%) had upper respiratory tract infection (URI) in the form of pharyngitis, four patients $(26.7 \%)$ had urinary tract infection (UTI) and four patients $(26.7 \%)$ had skin and soft tissue infections (3 with cellulitis and 1 with abscess). Nine patients had gram +ve organisms and six had gram -ve organisms.

Base line characteristics and comparison between studied groups are shown in (Table 1). All groups were matched regarding age, gender and BMI. There were significant differences among the studied groups regarding the CD64 expression percent and Mean fluorescence intensity (MFI) as well as serum PCT with $\mathrm{P}$ value $<0.05$. In between groups comparisons showed that in group I (control group) their levels were significantly lower than in the other three groups. Levels in group II (SLE group) were significantly lower than in groups III and IV. Finally levels in group III (SLE with activity), were significantly lower than in group IV (SLE with infection).

Table 1. Demographic and laboratory findings of the studied groups $(n=75)$.

\begin{tabular}{|c|c|c|c|c|c|}
\hline Variable & $\begin{array}{l}\text { Group I } \\
\text { Control } \\
(\mathbf{n}=\mathbf{2 0})\end{array}$ & $\begin{array}{l}\text { Group II } \\
\text { SLE without activity } \\
(\mathbf{n}=20)\end{array}$ & $\begin{array}{l}\text { Group III } \\
\text { SLE with activity } \\
(\mathbf{n}=\mathbf{2 0})\end{array}$ & $\begin{array}{l}\text { Group IV } \\
\text { SLE with infection } \\
(n=15)\end{array}$ & P value \\
\hline Age (years) & $34.35 \pm 8.43$ & $35.81 \pm 8.23$ & $33.55 \pm 10.31$ & $33.53 \pm 11.74$ & $>0.05$ \\
\hline BMI $\left(\mathrm{kg} / \mathrm{m}^{2}\right)$ & $25.74 \pm 2.34$ & $27.01 \pm 1.55$ & $28.60 \pm 4.35$ & $26.59 \pm 5.02$ & $>0.05$ \\
\hline TLC $(\times 1000)$ & $7.11 \pm 1.91$ & $7.93 \pm 2.36$ & $6.04 \pm 2.92$ & $19.85 \pm 7.07$ & $<0.05^{* * *, \# \#, \$}$ \\
\hline $\operatorname{ESR}(\mathrm{mm} / \mathrm{h})$ & $15.40 \pm 2.87$ & $26.43 \pm 9.37$ & $97.00 \pm 26.37$ & $87.33 \pm 16.89$ & $<\mathbf{0 . 0 5}$ \\
\hline CRP (mg/dl) & $6.90 \pm 2.19$ & $8.00 \pm 2.89$ & $26.70 \pm 13.83$ & $22.20 \pm 5.94$ & 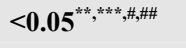 \\
\hline C3 (ng/ml) & $126.65 \pm 30.46$ & $129.43 \pm 30.65$ & $76.50 \pm 20.98$ & $129.00 \pm 19.66$ & $<0.05^{* *, \#, \$}$ \\
\hline C4 (ng/ml) & $23.75 \pm 10.62$ & $21.19 \pm 8.79$ & $10.30 \pm 3.12$ & $28.00 \pm 6.21$ & $<0.05^{* *, \#, \$}$ \\
\hline SLEDAI score & $0.0 \pm 0.0$ & $2.24 \pm 1.55$ & $21.60 \pm 3.99$ & $3.20 \pm 1.61$ & $<0.05^{*, * * * * * * \#, \mathbf{S}}$ \\
\hline N. count $(\times 1000)$ & $3.59 \pm 1.51$ & $4.36 \pm 1.67$ & $3.39 \pm 2.01$ & $15.82 \pm 7.45$ & $<0.05^{* * * \# \#, \$}$ \\
\hline N. percent & $49.25 \pm 8.92$ & $54.71 \pm 9.80$ & $55.25 \pm 9.23$ & $78.33 \pm 7.58$ & $<0.05^{* *, * * *, \# \#, S}$ \\
\hline CD64\% & $47.36 \pm 7.57$ & $58.59 \pm 6.83$ & $81.30 \pm 5.15$ & $91.14 \pm 5.04$ & $<0.05^{*, * *, * * *, \#, \# \#, \mathbf{S}}$ \\
\hline CD64 MFI & $33.13 \pm 4.26$ & $57.95 \pm 7.43$ & $180.02 \pm 44.01$ & $339.89 \pm 106.05$ & $<0.05^{*, * *, * * *, \#, \# \#, \mathbf{S}}$ \\
\hline Serum PCT (ng/ml) & $0.03 \pm 0.01$ & $0.05 \pm 0.02$ & $0.14 \pm .0 .07$ & $1.16 \pm .0 .77$ & $<0.05^{* * * * * *, \#, \# \#, \mathbf{S}}$ \\
\hline
\end{tabular}

*: Control VS SLE, **: Control VS SLE with activity, ***: Control VS SLE with infection, \#: SLE VS SLE with activity, \#\#: SLE VS SLE with infection, \$: SLE with activity VS SLE with infection, BMI: Body mass index, TLC: total leucocytic count, ESR: erythrocyte sedimentation rate, CRP: C reactive protein C3: complement component 3, C4: complement component 4, SLEDAI score: systemic lupus erythematosus disease activity index score, N. count: Neutrophil count, N. percent: Neutrophil percent, CD64: cluster of differentiation 64, MFI: Mean fluorescence intensity, Serum PCT: Procalcitonin

Pearson's correlations between CD64 expression and markers of inflammation and infection are shown in (Table 2). There were significantly positive correlations between CD64 expression and ESR $(\mathrm{r}=0.816, \mathrm{p}<0.05)$ and SLEDAI score $(\mathrm{r}$ $=0.340, \mathrm{P}<0.05)$, on the other hand a significantly negative correlation $(\mathrm{r}=-0.279, \mathrm{P}<0.05)$ was found only with $\mathrm{C} 3$ and not C4. Also, there were significant positive correlations between CD64 expression and CRP $(r=0.471, \mathrm{P}<0.05)$, TLC $(\mathrm{r}=0.469, \mathrm{p}<0.05)$, neutrophil percent $(\mathrm{r}=0.576, \mathrm{P}<0.05)$ and neutrophil count $(\mathrm{r}=0.508, \mathrm{p}<0.05)$.

Pearson's correlations between serum PCT and the same markers are shown in (Table 3). There were significantly positive correlations between serum PCT and ESR ( $\mathrm{r}=0.65$, $\mathrm{p}<0.05$ ) but no significant correlations were found with SLEDAI score, $\mathrm{C} 3$ or $\mathrm{C} 4$. On the other hand, there were significant positive correlations between serum PCT and 
CRP ( $\mathrm{r}=0.471, \mathrm{P}<0.05)$, TLC $(\mathrm{r}=0.45, \mathrm{p}<0.05)$, neutrophil percent $(\mathrm{r}=0.65, \mathrm{p}<0.05)$ and neutrophil count $(\mathrm{r}=0.51$, $\mathrm{p}<0.05)$.

Table 2. Correlations between neutrophil CD64 expression and other markers in SLE patients $(n=55)$.

\begin{tabular}{lll}
\hline Variable & $(\mathbf{r})$ & P value \\
\hline ESR & 0.816 & $<\mathbf{0 . 0 5}$ \\
CRP & 0.471 & $<\mathbf{0 . 0 5}^{*}$ \\
C3 & -0.279 & $<\mathbf{0 . 0 5}^{*}$ \\
C4 & 0.077 & $>0.05$ \\
SLEDAI score & 0.340 & $<0.05^{*}$ \\
TLC & 0.469 & $<\mathbf{0 . 0 5}^{*}$ \\
N. count & 0.576 & $<\mathbf{0 . 0 5}^{*}$ \\
N. percent & 0.508 & $<\mathbf{0 . 0 5}^{*}$ \\
\hline
\end{tabular}

$* \mathrm{P}<0.05$ : significant, CD64: cluster of differentiation 64, ESR: erythrocyte sedimentation rate, CRP: $\mathrm{C}$ reactive protein $\mathbf{C 3}$ : complement component 3, C4: complement component 4, SLEDAI score: systemic lupus erythematosus disease activity index score, TLC: total leucocytic count, $\mathbf{N}$. count: Neutrophil count, $\mathbf{N}$. percent: Neutrophil percent

Table 3. Correlations between serum PCT and other markers in SLE patients $(n=55)$.

\begin{tabular}{lll}
\hline Variable & $(\mathbf{r})$ & P value \\
\hline ESR & 0.65 & $<0.05^{*}$ \\
CRP & 0.471 & $<0.05^{*}$ \\
C3 & -0.06 & $>0.05$ \\
C4 & 0.17 & $>0.05$ \\
SLEDAI score & 0.18 & $>0.05$ \\
TLC & 0.45 & $<0.05^{*}$ \\
N. count & 0.65 & $<0.05^{*}$ \\
N. percent & 0.51 & $<0.05^{*}$ \\
\hline
\end{tabular}

*P<0.05: significant, Serum PCT: Procalcitonin, ESR: erythrocyte sedimentation rate, CRP: C reactive protein, C3: complement component 3, C4: complement component 4, SLEDAI score: systemic lupus erythematosus disease activity index score, TLC: total leucocytic count, $\mathbf{N}$. count: Neutrophil count, N. percent: Neutrophil percent

We performed ROC curves using neutrophil CD64 expression and serum PCT for detecting infection (i.e.

$$
r=0.40 \quad P \text { value }=0.002
$$

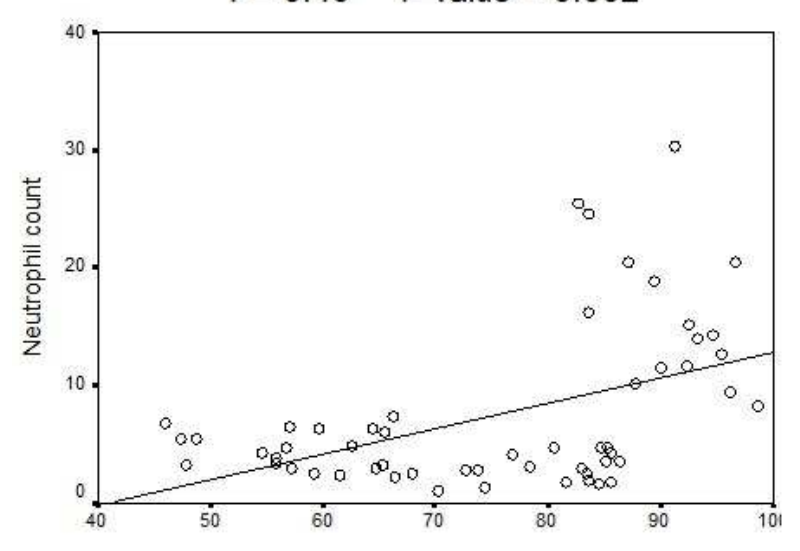

$\operatorname{CD} 64$ between groups 3 and 4). The area under the ROC curves (AUC; 95\% CI) for neutrophil CD64 expression and serum PCT were $(0.90 ; 0.79-1.01)$ and $(0.99 ; 0.95-1.01)$, respectively, which were significant $(\mathrm{P}<0.05)$. The best cut off value for CD64 was $82.11 \%$, with a sensitivity of $80 \%$ and a specificity of $95 \%$. The best cut off value for PCT was $0.23 \mathrm{ng} / \mathrm{mL}$, with a sensitivity of $100 \%$ and a specificity of $80 \%$. The predicted accuracy for CD64 and PCT combined was $91.4 \%$, with a sensitivity of $100 \%$ and a specificity of $85 \%$. (Tables 4 and 5 and Figure 3)

Table 4. Cut off levels of CD64 expression, serum PCT for differentiating infection from activity in SLE patients (between groups III and IV).

\begin{tabular}{lll}
\hline Variable & CD64\% & Serum PCT \\
\hline AUC & 0.90 & 0.99 \\
P VALUE & $<0.001$ & $<0.001$ \\
$95 \%$ CI & $0.79-1.01$ & $0.98-1.01$ \\
Cutoff point & 86.75 & 0.23 \\
\hline
\end{tabular}

Serum PCT: Procalcitonin, AUC: area under the curve, CI: Confidence interval

Table 5. Validity of the individual and combined tests of CD64 expression and serum PCT for differentiating infection from activity in SLE patients (between groups III and IV).

\begin{tabular}{llll}
\hline Variable & CD64\% & Serum PCT & Combined \\
\hline Sensitivity & $80 \%$ & $100 \%$ & $100 \%$ \\
Specificity & $95 \%$ & $90 \%$ & $85 \%$ \\
PPV & 92.3 & 88.2 & $83.3 \%$ \\
NPP & 86.4 & 100 & $100 \%$ \\
Accuracy & 88.6 & 94.3 & $91.4 \%$ \\
\hline
\end{tabular}

Serum PCT: Procalcitonin, PPV: Positive predictive value, NPP: Negative predictive value

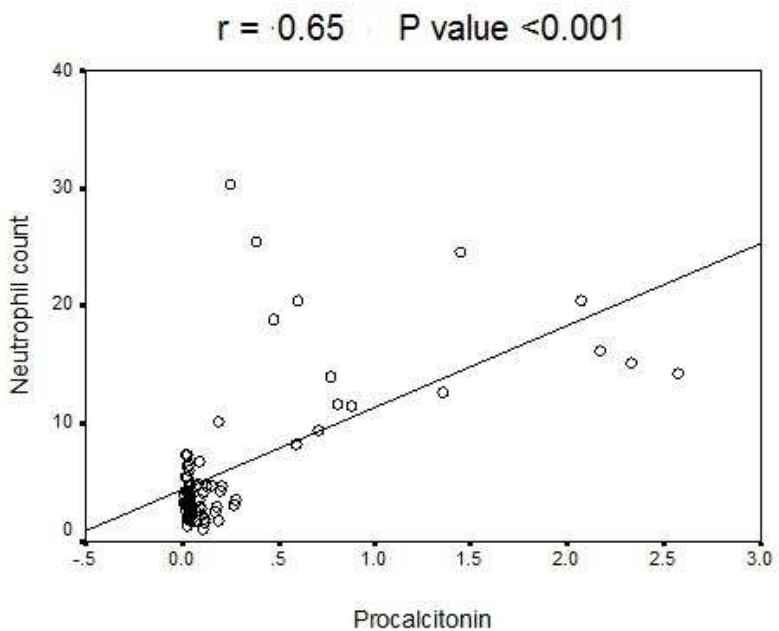

Figure 1. Correlations between CD64 expression, serum PCT and neutrophil count in SLE patients ( $n=55)$. 

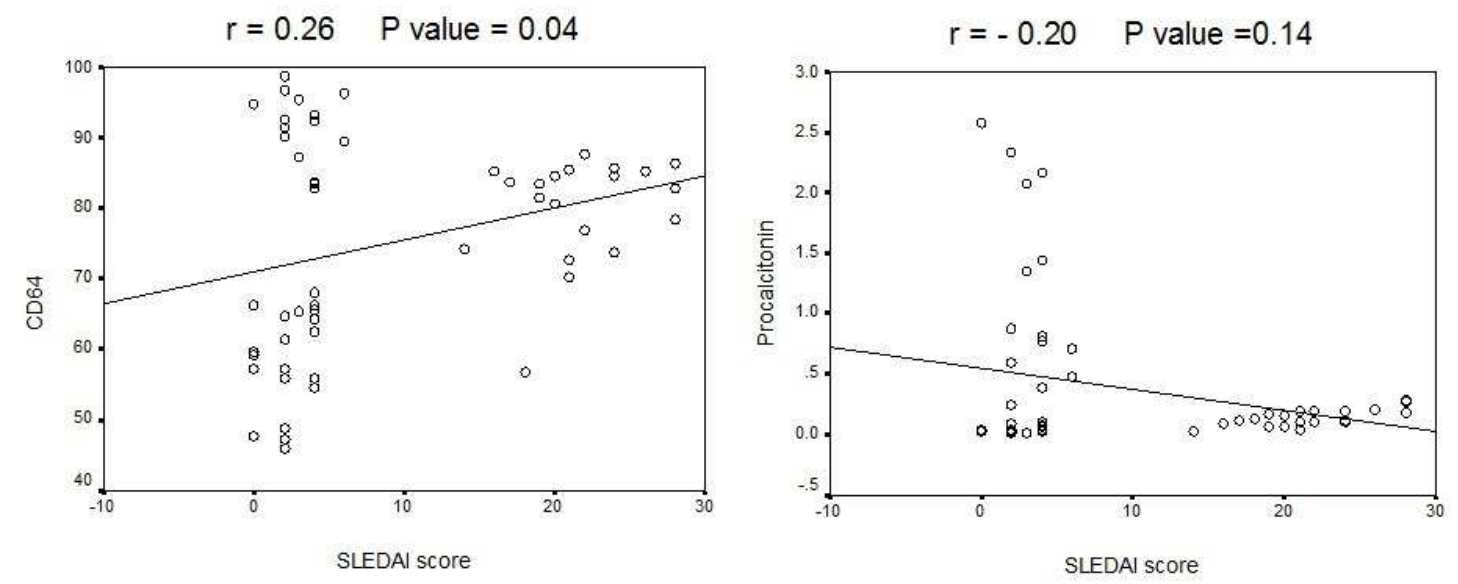

Figure 2. Correlations between CD64 expression, serum PCT and SLEDAI score in SLE patients ( $n=55)$.

\section{ROC Curve}

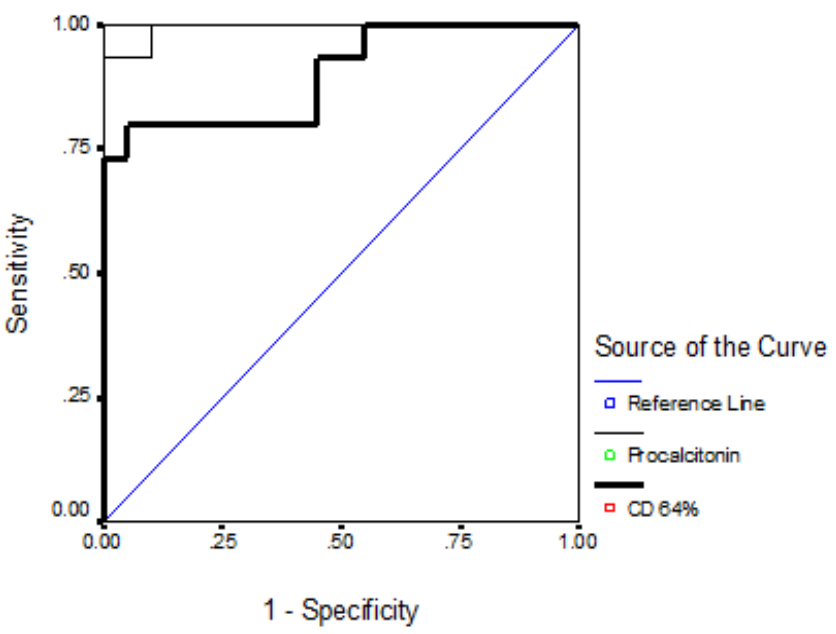

Figure 3. ROC curves of CD64 expression and serum PCT for differentiating infection from activity in SLE patients (between groups III and $I V)$.

\section{Discussion}

Infection is responsible for approximately $25 \%$ of all deaths in patients with SLE, making it a leading cause of mortality among those patients [23]. In autoimmune inflammatory diseases, infection is often hard to be detected because fever, malaise and other constitutional symptoms do not enable physicians to distinguish infections from a flare of the underlying disease [3]. The usual laboratory tests can be misleadingly low in patients with systemic infections who are receiving corticosteroid or cytotoxic treatment. On the other hand, they may be misleadingly high in those with active rheumatic diseases. A positive culture usually requires 24 hours or longer before results can be obtained [24]. In clinical practice, due to the major differences in treating the two different situations, flare or infection, a rapid diagnosis is required to be helpful in the difficult decision as to start or increase immunosuppresion or to start antibacterial therapy and reduce immunosuppresion if needed.

One of the most useful applications of CD64 is probably its ability to distinguish between bacterial infection and acute flares in autoimmune disorders [25].

Similarly, expression of serum procalcitonin is not elevated following non-infectious inflammation or nonbacterial infections [26], so both markers may be useful as markers to distinguish bacterial infection from disease flare in patients with SLE. In the current work, we aimed to study the usefulness of both neutrophil CD64 expression and serum PCT as diagnostic markers to discriminate infection from disease activity in SLE patients.

In this study, CD64 expression and MFI levels were highest in the infection group compared to the first three groups. Their levels in the activity group were higher than in the second group which in turn were higher than the control group. Hussein and coworkers studied CD64 expression and MFI in SLE patients with activity and with bacterial infections. They found that in the infection group, CD64 expression and MFI were significantly higher than in the active inflammatory group [13]. These results are similar to the results of Allen and coworkers who found that in autoimmune disease, neutrophil CD64 expression was greater in systemic infections than in non-inflammatory and inflammatory disease [27]. These results are much like ours except that we found a significant difference between controls and SLE patients without activity.

We also found a similar pattern of changes in serum PCT levels which were highest in the infection group compared to the first three groups. Their levels in the activity group were higher than in the first two groups with no significant difference between them. These results are consistent with the results obtained by Tamaki and colleagues who measured serum PCT in patients with systemic autoimmune disease presented with either disease activity or bacterial infections. They found that serum PCT levels were higher in the infection group than the disease activity group [8]. Also, Joo and colleagues found that serum PCT levels in patients with autoimmune diseases were higher in the bacterial infection group than in the disease flare group [9].

In the current work, we performed Pearson's correlations between CD64 expression and the laboratory markers of inflammation, activity and infection. Significant correlations 
were found between CD64 expression and markers of inflammation and disease activity, i.e. significantly positive with ESR and SLEDAI score and negative with C3. On the other hand significant positive correlations were found with the markers of infection, i.e. CRP, TLC, neutrophil count and percent. Consistent with these observations, Hussein and colleagues found significant positive correlations between CD64 expression and SLEDAI score. Also, significant positive correlations were found with the TLC, and neutrophil percentage. However unlike our results, no significant correlations were found with ESR, CRP or neutrophil count [13]. On the other hand, Goulding and coworkers studied CD64, CD32 and CD16 expressions on neutrophil purified from autoimmune patients' peripheral blood. CD64 expression on the peripheral blood neutrophils was within normal limits in patients with active RA but increased CD64 bearing neutrophils in the synovial fluid. They concluded that the difference in synovial neutrophil activation might be either due to intra-articular cytokines or other factors as immune complexes [28]. While Szucs and colleagues reported that CD64 expression on the peripheral blood neutrophils in patients with SLE was not up regulated, although the activity of SLE was not mentioned in that study [29].

Similarly, we performed Pearson's correlations between serum PCT and the laboratory markers of inflammation, activity and infection. Unlike CD64, serum PCT was significantly correlated with ESR but not with markers of disease activity, i.e. complement components or SLEDAI score. However, there were significant positive correlations with markers of infections, i.e. CRP, TLC, neutrophil count and percent. So, unlike CD64 which was affected with both infection and disease activity, serum PCT was more affected with infection than with disease activity.

We demonstrated ROC curves of both markers for differentiation between infection and activity in SLE patients. Hussein and coworkers stated that, a level of CD64 $\geq 43.5 \%$ was found to be both sensitive and specific (94.4\%, 88.9\% respectively) for detection of infection in patients with autoimmune inflammatory diseases [13]. Allen and colleagues confirmed the high sensitivity and specificity of CD64 expression on neutrophils to differentiate systemic infection from active inflammatory autoimmune diseases; those were $85 \%$ and $91 \%$ respectively [27]. In this work, we demonstrated that neutrophil CD64 $\geq 82.11 \%$ was found to have a sensitivity of $80 \%$ and a specificity of $95 \%$ in detecting infection.

Also, a number of studies demonstrated different cut off levels of serum PCT to detect infection in patients with autoimmune diseases [7-9, 30-34]. Wu and colleagues performed a meta-analysis of nine studies that tested the value of serum PCT as a marker for differentiating bacterial infection from disease flare in febrile patients with autoimmune disease [26]. They concluded that the area under the ROC curve (AUC; 95\% CI) for serum PCT was $(0.91$; 0.88-0.931). Six of these studies used a cut off level of 0.5 $\mathrm{ng} / \mathrm{mL}$, two used lower levels $(0.09$ and $0.3 \mathrm{ng} / \mathrm{mL})$ and one demonstrated a higher level $1.4 \mathrm{ng} / \mathrm{mL}$. In the current study, the area under the ROC curve (AUC; 95\% CI) for serum PCT was $(0.99 ; 0.95-1.01)$, with the best cut off value was $0.23 \mathrm{ng} / \mathrm{mL}$.

In this work, serum PCT was found to be more accurate than neutrophil CD64 in detecting infection with AUC of 0.90 and 0.99 respectively. Serum PCT was also found to have a higher sensitivity but a lower specificity when compared with neutrophil CD64. The accuracy of both markers together has not been tested before for detection of infection in patients with autoimmune disorders. However, Cardelli and colleague found that CD64 had a better specificity when compared with PCT for diagnosing sepsis in ICU patients [35]. In another study PCT was compared with CD64 and soluble triggering receptor expressed on myeloid cells-1 (sTREM-1) in diagnosis of infection in critically ill patients, the best ROC curve was obtained by using CD64 [36]. Finally, Zeitoun and coworkers used PCT, neutrophil CD64 and Interleukin 10 (IL-10) for diagnosis of neonatal sepsis. They found that neutrophil CD64 had a better sensitivity and specificity when compared to PCT [37]. The possible differences between our results and the previously mentioned results could be explained by the difference in the type of patients enrolled in our study as the inflammatory state in SLE patients with activity also affects neutrophil CD64 expression [13].

\section{Conclusion}

The previous findings can prove that both neutrophil CD64 expression and serum PCT are reliable markers for diagnosis of infection in SLE patients. Serum PCT was more accurate than neutrophil CD64 expression. The use of the two markers enhanced the specificity with no effect on the sensitivity of PCT. Further more comprehensive studies are warranted to estimate reliable cut off levels that can be used clinically for the diagnosis of infection in SLE patients.

\section{References}

[1] Marian V, Anolik JH. Treatment targets in systemic lupus erythematosus: biology and clinical perspective. Arthritis Research \& Therapy 2012; 14:S3.

[2] Davis BH. Improved diagnostic approaches to infection/sepsis detection. Expert Rev Mol Diagn 2005; 5:193-207.

[3] Doi T, Miyazaki T, Nishino J, Tanaka S, Matsui T, Komiya A et al. Neutrophil CD64 expression as a diagnostic marker for local infection and crystal-induced arthritis. Mod Rheumatol 2010; 20(6):573-9.

[4] Livaditi O, Kotanidou A, Psarra A, Dimopoulou I, Sotiropoulou C, Augustatou K et al. Neutrophil CD64 expression and serum IL-8: sensitive early markers of severity and outcome in sepsis. Cytokine 2006; 36:283-90.

[5] Hoffmann J. Neutrophil CD64 as a sepsis biomarker. Biochemia Medica 2011; 21:282-90. 
[6] Assicot M, Gendrel D, Carsin H, Raymond J, Guilbaud J, Bohuon C. High serum procalcitonin concentrations in patients with sepsis and infection. Lancet 1993; 341:515-8.

[7] Suzuki N, Mizuno H, Nezu M, Takai Y, Misu T, Kuroda H, et al. Procalcitonin might help in discrimination between meningeal neuro-Behcet disease and bacterial meningitis. Neurology 2009; 72:762-3.

[8] Tamaki K, Kogata Y, Sugiyama D, Nakazawa T, Hatachi S, Kageyama G, et al. Diagnostic accuracy of serum procalcitonin concentrations for detecting systemic bacterial infection in patients with systemic autoimmune diseases. J Rheumatol 2008; 35:114-9.

[9] Joo K, Park W, Lim MJ, Kwon SR, Yoon J. serum procalcitonin for differentiating bacterial infection from disease flares in patients with autoimmune diseases. J Korean Med Sci 2011; 26:1147-51.

[10] Tan EM, Cohen AS, Fries JF, Masi AT, McShane DJ, Rothfield NF et al. The 1982 revised criteria for the classification of systemic lupus erythematosus. Arthritis Rheum 1982; 25(11):1271-7.

[11] Hochberg MC. Updating the American College of Rheumatology revised criteria for the classification of systemic lupus erythematosus. Arthritis Rheum 1997; 40:1725 .

[12] Gladman DD, Ibanez D, Urowitz MB. Systemic Lupus Erythematosus Disease Activity Index 2000. J Rheumatol 2002; 29:288-91.

[13] Hussein OA, El-Toukhy MA, and El-Rahman HS. Neutrophil CD64 Expression in Inflammatory Autoimmune Diseases: Its Value in Distinguishing Infection from Disease Flare. Immunol Invest 2010; 39:699-712.

[14] Liu S, Ren J, Xia Q, Wu X, Han G, Ren H et al. Preliminary Case-control Study to Evaluate Diagnostic Values of CReactive Protein and Erythrocyte Sedimentation Rate in Differentiating Active Crohn's Disease From Intestinal Lymphoma, Intestinal Tuberculosis and Behcet's Syndrome. Am j med sci 2013; 346 (6): 467-72.

[15] Hindocha P, Campbell CA, Gould JD, Wojciechowski A, Wood CB. Sequential study of C reactive protein in neonatal septicaemia using a latex agglutination test. J Clin Pathol 1984; 37(9):1014-7.

[16] Homburger HA, Cahen YD, Griffiths J, Jacob G. Detection of antinuclear antibodies: Comparative evaluation of enzyme immunoassay and indirect immunofluorescence methods. Arch of Pathol Lab Med 1998; 122(11): 993-9.

[17] Stokes RP, Cordwell A and Thompson RA; A simple, rapid ELISA method for detection of DNA antibodies. J Clin Pathol 1982; 35: 566-73.

[18] Frederikson GN, Truedsson L and Sjoeholm AG. New procedure for detection of complement deficiency by ELISA, analysis of activation pathway and circumvention of rheumatoid factor influence. J Immunol Methods 1993; 166(2): 263-70.

[19] Brown M, Wittwer C. Flow cytometry: principles and clinical applications in hematology. Clin Chem. 2000; 46:1221-9.
[20] Deneys V, Michaux L, Leveugle P, Mazzon AM, Gillis E, Ferrant A et al. Atypical lymphocytic leukemia and mantle cell lymphoma immunologically very close: flow cytometric distinction by the use of CD20 and CD54 expression. Leukemia 2001; 15(9):1458-65.

[21] Schuetz P, Briel M, Christ-Crain M, Stolz D, Bouadma L, Wolff $\mathrm{M}$ et al. Procalcitonin to Guide Initiation and Duration of Antibiotic Treatment in Acute Respiratory Infections: An Individual Patient Data Meta-Analysis. Clin Infect Dis 2012; 55(5):651-62.

[22] Kopterides P, Siempos, II, Tsangaris I, Tsantes A and Armaganidis A. Procalcitonin-guided algorithms of antibiotic therapy in the intensive care unit:a systematic review and metaanalysis of randomized controlled trials. Crit Care Med 2010; 38(11): 2229-41.

[23] Goldblatt F, Chambers S, Rahman A, Isenberg DA. Serious infections in British patients with systemic lupus erythematosus: hospitalisations and mortality. Lupus 2009; 18(8):682-9.

[24] Jalava-Karvinen P, Hohenthal U, Laitinen I, Kotilainen P, Rajamäki A, Nikoskelainen J, et al. Simultaneous quantitative analysis of Fc gamma RI (CD64) and CR1 (CD35) on neutrophils in distinguishing between bacterial infections, viral infections, and inflammatory diseases. Clin Immunol 2009; 133:314-323.

[25] Qureshi SS, Lewis SM, Gant VA, Treacher D, Davis BH, Brown KA. Increased distribution and expression of CD64 on blood polymorphonuclear cells from patients with the systemic inflammatory response syndrome (SIRS). Clin Exp Immunol 2001; 125:258-65.

[26] Wu JY, Lee SH, Shen CJ, Hsieh YC, Yo PH, Cheng HY, et al. Use of Serum PCT to Detect Bacterial Infection in Patients with Autoimmune Diseases. A Systemic Review and MetaAnalysis. Arthritis Rheum 2012; 64 (9): 3034-42.

[27] Allen E, Bakke AC, Purtze MZ, Deodhar A. Neutrophil CD64 expression distinguishing acute inflammatory disease from systemic infections. Ann Rheum Dis 2002; 61(6):522-5.

[28] Goulding NJ, Knight SM, Godolphin JL, Guyre PM. Increase in neutrophil Fc gamma receptor I expression following interferon gamma treatment in rheumatoid arthritis. Ann Rheum Dis 1992; 51(4):465-8.

[29] Szucs G, Kavai M, Kiss E. Correlation of IgG Fc receptors on granulocytes with serum immune complex level insystemic lupus erythrematosus. Scand J Immunol 1995; 42:577-80.

[30] Brunkhorst R, Eberhardt OK, Haubitz M, Brunkhorst FM. Procalcitonin for discrimination between activity of systemic autoimmune disease and systemic bacterial infection. Intensive Care Med 2000; 26 (2):199-201.

[31] Chen DY, Chen YM, Ho WL, Chen HH, Shen GH, Lan JL. Diagnostic value of procalcitonin for differentiation between bacterial infection and non-infectious inflammation in febrile patients with active adult-onset Still's disease. Ann Rheum Dis 2009; 68:1074-5.

[32] Eberhard OK, Haubitz M, Brunkhorst FM, Kliem V, Koch $\mathrm{KM}$, Brunkhorst R. Usefulness of procalcitonin for differentiation between activity of systemic autoimmune disease (systemic lupus erythematosus/systemic antineutrophil cytoplasmic antibody-associated vasculitis) and invasive bacterial infection. Arthritis Rheum 1997; 40:1250-6. 
[33] Martinot M, Sordet C, Soubrier M, Puechal X, Saraux A, Liote $\mathrm{F}$, et al. Diagnostic value of serum and synovial procalcitonin in acute arthritis: a prospective study of 42 patients. Clin Exp Rheumatol 2005; 23:303-10.

[34] Scire CA, Cavagna L, Perotti C, Bruschi E, Caporali R, Montecucco C. Diagnostic value of procalcitonin measurement in febrile patients with systemic autoimmune diseases. Clin Exp Rheumatol 2006; 24:123-8.

[35] Cardelli P1, Ferraironi M, Amodeo R, Tabacco F, De Blasi RA, Nicoletti M, et al. Evaluation of neutrophil CD64 expression and procalcitonin as useful markers in early diagnosis of sepsis. Int J Immunopathol Pharmacol. 2008; 21(1):43-9.
[36] Gibot S, Béné MC, Noel R, Massin F, Guy J, Cravoisy A, et al Combination biomarkers to diagnose sepsis in the critically ill patient. Am J Respir Crit Care Med. 2012; 186 (1):65-71.

[37] Zeitoun AA, Gad SS, Attia FM, Abu Maziad AS, Bell EF. Evaluation of neutrophilic CD64, interleukin 10 and procalcitonin as diagnostic markers of early- and late-onset neonatal sepsis. Scand J Infect Dis. 2010; 42 (4):299-305. 